\title{
Side Chain Orientation of the Amino Acid Substituted by a Cysteine Residue Is Important for Successful Crosslinking of Galectin to Its Glycoprotein Ligand Using a Photoactivatable Sulfhydryl Reagent
}

\author{
Mayumi TAmURA, ${ }^{a}$ Takanori IgARASHI,${ }^{b}$ Ken-ichi KASAI,${ }^{b}$ and Yoichiro ARATA ${ }^{*, a}$ \\ ${ }^{a}$ Laboratory of Molecular Immunology, Faculty of Pharmaceutical Sciences, Josai University, \\ 1-1 Keyakidai, Sakado, Saitama 350-0295, Japan, and bepartment of Biological \\ Chemistry, Faculty of Pharmaceutical Sciences, Teikyo University, 1091-1 \\ Suarashi, Midori-ku, Sagamihara, Kanagawa 252-5195, Japan
}

(Received March 4, 2010; Accepted June 26, 2010)

\begin{abstract}
We have employed a combination of cysteine mutagenesis and chemical crosslinking using a photoactivatable sulfhydryl reagent, benzophenone-4-maleimide, to obtain a covalent complex between human galectin-1 and a model glycoprotein ligand, asialofetuin. We previously obtained a crosslinked product when Lys ${ }^{28}$ of the cysteine-less form of human galectin-1 was mutated to cysteine. To investigate whether substituting either of the two flanking amino acid residues in the same $\beta$-strand, $\mathrm{Ala}^{27}$ and $\mathrm{Ser}^{29}$, to cysteine could result in crosslinking to the bound asialofetuin, two cysteine-containing mutants were generated. Although both the mutants adsorbed to asialofetuin-agarose and were eluted with $0.1 \mathrm{M}$ lactose, confirming their ability to interact with asialofetuin, these mutants did not crosslink to the bound glycoprotein ligand following treatment with benzophenone-4-maleimide. Therefore the orientation of the side chain of the introduced cysteine residue apparently plays an important role in the crosslinking reaction.
\end{abstract}

Key words_- galectin; asialofetuin; crosslink; ligand; benzophenone-4-maleimide

\section{INTRODUCTION}

Galectins are a group of animal lectins characterized by their specificity for $\beta$-galactosides and by an evolutionarily conserved sequence motif in their carbohydrate binding site. Galectins are involved in a wide variety of biological phenomena, including development, cell differentiation, tumor metastasis, apoptosis, RNA splicing, and regulation of immune function. ${ }^{1-6)}$

Interactions between lectins and their carbohydrate ligands play important roles in various biological systems. The relatively weak binding of lectins to their ligands allows more frequent binding and dissociation. Unfortunately, weak interactions can hinder identification of physiological ligands using conventional methods such as affinity purification. Although quite a few candidate galectin ligands have been identified to date, ${ }^{7)}$ considering the variety of sugar-binding specificity among galectins, ${ }^{8,9)}$ it is likely that other ligands remain to be identified.

We have previously employed mutants of the nematode tandem repeat-type galectin, LEC-1, ${ }^{10}$ )

\footnotetext{
*e-mail: arata@josai.ac.jp
}

which contains a single cysteine residue, for chemical crosslinking using the photoactivatable sulfhydryl reagent, benzophenone-4-maleimide (BPM)..11-13) These mutants were used to detect the interaction between $N$-acetyllactosamine-containing glycoprotein and the cysteine-introduced galectin. Among the five LEC-1 mutants containing a unique cysteine residue at $\mathrm{Arg}^{14}$ (R14C), $\mathrm{Gln}^{38}$ (Q38C), $\mathrm{Thr}^{41}$ (T41C), $\mathrm{Asp}^{50}$ (D50C), or Gly ${ }^{53}$ (G53C), only one of them (Q38C) resulted in the crosslinked product between LEC-1 and asialofetuin via BPM.10) This result suggests that the position of the Cys in LEC-1 is important for successful crosslinking.

We applied this method to human Gal-1 so as to establish a methodology for generating a covalent complex between Gal-1 and potential novel ligands, especially those with relatively low affinity for Gal-1. Lys $^{28}$ in human Gal-1 (Cys-less form), which is at a location that corresponds to $\mathrm{Gln}^{38}$ of LEC-1, was mutated to Cys (K28C) and the mutant was crosslinked to asialofetuin using BPM. ${ }^{14)}$ To confirm that the extension of the substituted cysteine side chain towards the bound glycoprotein ligand plays an important role in crosslinking, we created two more Cys-containing mutants of the Cys-less form of human Gal-1. Ac- 
cording to the X-ray crystallographic structure of human Gal-1, ${ }^{15)} \mathrm{Ala}^{27}$ and $\mathrm{Ser}^{29}$ flank $\mathrm{Lys}^{28}$ in the $\beta$ strand. These two amino acids, $\mathrm{Ala}^{27}$ and $\mathrm{Ser}^{29}$, were substituted by Cys (A27C and S29C, respectively) and tested to determine whether these two mutants could crosslink to asialofetuin via BPM. Although both of the mutants adsorbed to asialofetuin-agarose and eluted with $0.1 \mathrm{M}$ lactose, confirming their ability to interact with asialofetuin, they did not crosslink to the bound asialofetuin following reaction with BPM. Therefore extension of the side chain of the introduced cysteine residue (which provides the sulfhydryl group that reacts with the maleimide in BPM) towards the bound glycoprotein ligand apparently plays an important role in crosslinking.

\section{MATERIALS AND METHODS}

Materials Rabbit anti-bovine fetuin was purchased from Chemicon International (Temecula, CA, USA). BPM and fetuin from fetal calf serum were purchased from Sigma (St Louis, MO, USA). Rabbit anti-human Gal-1 antibody was prepared as described previously. ${ }^{16)}$ Sialidase from Clostridium perfringens was purchased from New England BioLabs (Ipswich, MA, USA). Sephadex G-75 and a HiTrap NHS-activated-HP column were purchased from GE Healthcare (Buckinghamshire, England). Amicon Ultra-15 centrifugal filter units $(10,000$ NMWL) were purchased from Millipore (Billerica, MA, USA) .

Construction of K28C Mutant of Human Gal-1

Construction and expression of the recombinant K28C mutant of human Gal-1 were performed as described previously. ${ }^{14}$

Construction of A27C and S29C Mutants of Human Gal-1 Site-directed mutants were constructed using the Altered Sites II in vitro mutagenesis system (Promega) as described previously. ${ }^{10,14)}$ The following oligonucleotide primers were used for mutagenesis of the Cys-less form of Gal-1 cDNA to introduce a unique cysteine (underlined) at position $\mathrm{Ala}^{27}$ (A27C) or $\operatorname{Ser}^{29}$ (S29C).

A27C, 5'-AGGCGAGGTGGCGCCTGACTGTA AGAGCTTCGT-3' and S29C, 5'-CCTGACGCTA AGTGCTTCGTTTTAAACCTGGGCAAAGA-3' .

After the mutagenesis reaction, the NdeI/BamHI fragments of the mutant forms of Gal-1 cDNA (A27C and S29C) were ligated to the pET21a expression vector for protein expression. The sequences of all mutants were confirmed by DNA sequencing.

Purification of Recombinant Proteins The expression of recombinant proteins was performed as described previously. ${ }^{10,14,17)}$ The supernatant of sonicated Escherichia coli strain BL21 (DE3) transformed with pET21a-A27C or S29C was applied to an asialofetuin-Sepharose 4B column [column volume, $10 \mathrm{ml}$; $9.1 \mathrm{mg}$ protein $/ \mathrm{ml}$ gel prepared according to the method of de Waard et al. ${ }^{18)}$ ] that had been previously equilibrated with EDTA-ME-PBS $(20 \mathrm{mM}$ Naphosphate, pH 7.2, containing $150 \mathrm{mM} \mathrm{NaCl}, 4 \mathrm{mM}$ $\beta$-mercaptoethanol and $2 \mathrm{mM}$ EDTA). After washing the column with this buffer, the adsorbed proteins were eluted with $0.1 \mathrm{M}$ lactose in EDTA-ME-PBS.

Sialidase Treatment of Commercially Available Fetuin to Obtain Asialofetuin Bovine fetuin (100 $\mathrm{mg}, 20 \mathrm{mg} / \mathrm{ml}$ ) was treated with $250 \mathrm{U}$ sialidase at 37 ${ }^{\circ} \mathrm{C}$ overnight to remove the sialic acid attached to the nonreducing end of the sugar chain.

Gel Filtration of Asialofetuin Fraction Using Sephadex G-75 One milliliter of the sialidasetreated fetuin fraction was applied to a Sephadex G-75 column ( $14 \mathrm{~mm}$ i.d., column length $590 \mathrm{~mm}$, bed volume $91 \mathrm{ml}$ ) using phosphate-buffered saline containing 1 mM EDTA (EDTA-PBS, pH 7.2) as eluent. Fractions $(2 \mathrm{ml})$ were collected and protein concentrations measured by absorbance at $280 \mathrm{~nm}$. The presence of asialofetuin in each fraction was checked by western blotting using anti-fetuin antibody.

Affinity Purification of Asialofetuin Fraction Using an Immobilized LEC-1 Column Purified recombinant nematode LEC-1 was immobilized on a HiTrap NHS-activated Sepharose column following the manufacturer's protocol using $0.1 \mathrm{M} \mathrm{NaHCO}$ containing $0.1 \mathrm{M}$ lactose. Fractions containing asialofetuin were applied to the LEC-1 column (13 mg of LEC-1 immobilized on a 1-ml column bed) and after extensive washing, bound asialofetuin was eluted using $0.1 \mathrm{M}$ lactose. The presence and purity of asialofetuin was checked by western blotting of each fraction using anti-fetuin antibody.

Covalent Crosslinking of Mutated Human Gal-1 Protein (A27C, K28C, and S29C) to Purified Asialofetuin Purified recombinant mutant proteins were dialyzed against EDTA-PBS. Covalent crosslinking reactions were conducted as described previously. ${ }^{10,14)}$

SDS-PAGE and Western Blotting For analysis of crosslinked products, the proteins were separated 
by SDS-polyacrylamide gel electrophoresis (PAGE) and subjected to western blotting using a rabbit polyclonal antibody raised against human Gal-1 ${ }^{16)}$ and rabbit polyclonal antibody raised against fetuin.

\section{RESULTS AND DISCUSSION}

To purify the recombinant cysteine mutants of human Gal-1 and to assess the effects of cysteine mutagenesis, E. coli lysates containing the expressed recombinant proteins were applied to an asialofetuinSepharose 4B column and the column was washed with buffer, then the specifically adsorbed recombinant proteins were eluted with $0.1 \mathrm{M}$ lactose. Protein elution was monitored by measuring the protein concentration of each fraction. The elution profiles of

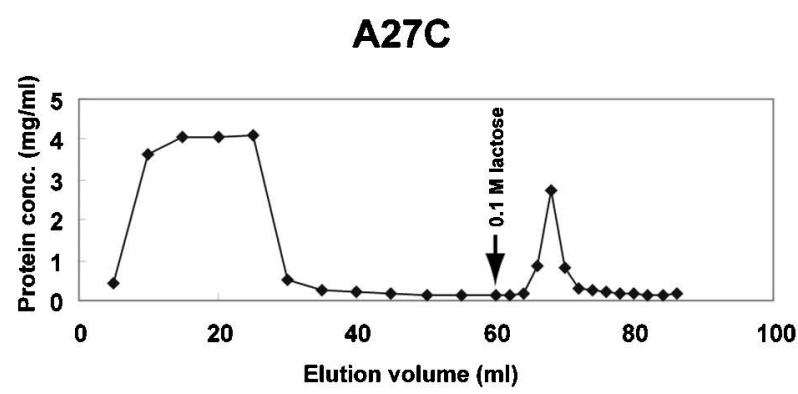

K28C

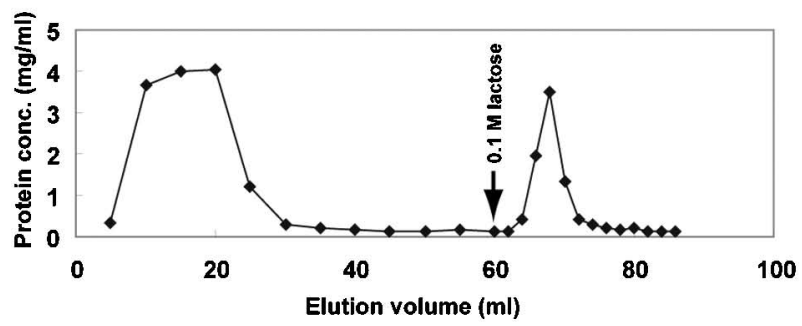

S29C

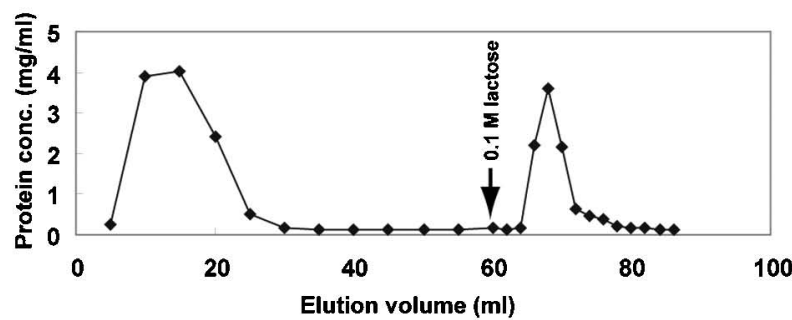

Fig. 1. Purification of Recombinant A27C, K28C, and S29C Gal-1

Elution profiles of recombinant A27C, K28C, and S29C Gal-1. E. coli extracts were applied to asialofetuin-Sepharose 4B columns. After extensive washing of the column with EDTA-ME-PBS, adsorbed mutant galectins were eluted with EDTA-ME-PBS containing $0.1 \mathrm{M}$ lactose. Fraction volumes: $5 \mathrm{ml}$ during washing; $2 \mathrm{ml}$ during elution with lactose. the A27C, K28C, and S29C mutants of human Gal-1 are shown in Fig. 1. Each of these purified mutant proteins gave a single band on SDS-PAGE (data not shown). Since the elution pattern of the three mutant proteins was basically the same as that of the Cys-less and $\mathrm{C} 2 \mathrm{~S}$ form of Gal-1, it can be concluded that each of the three cysteine-containing mutants of Gal-1 retained the ability to bind asialofetuin.

To ensure that the asialofetuin used as a model glycoprotein ligand for crosslinking to Gal-1 did not contain high-molecular weight proteins that could

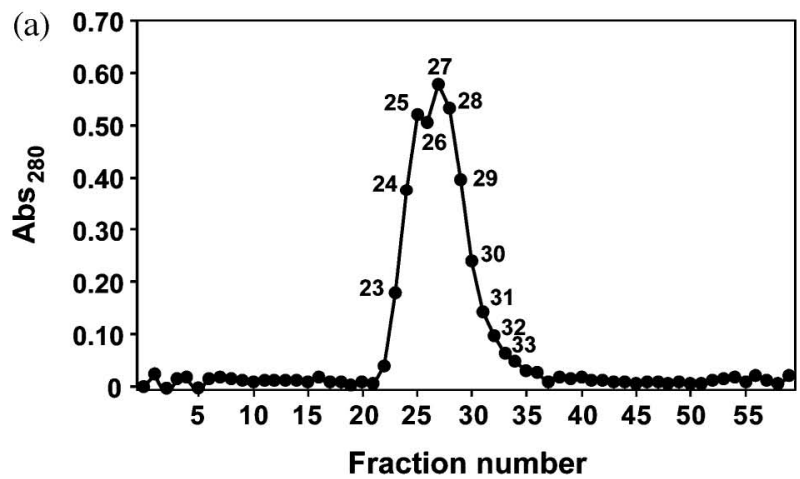

(b) $\begin{array}{lllllllllll}23 & 24 & 25 & 26 & 27 & 28 & 29 & 30 & 31 & 32 & 33\end{array}$

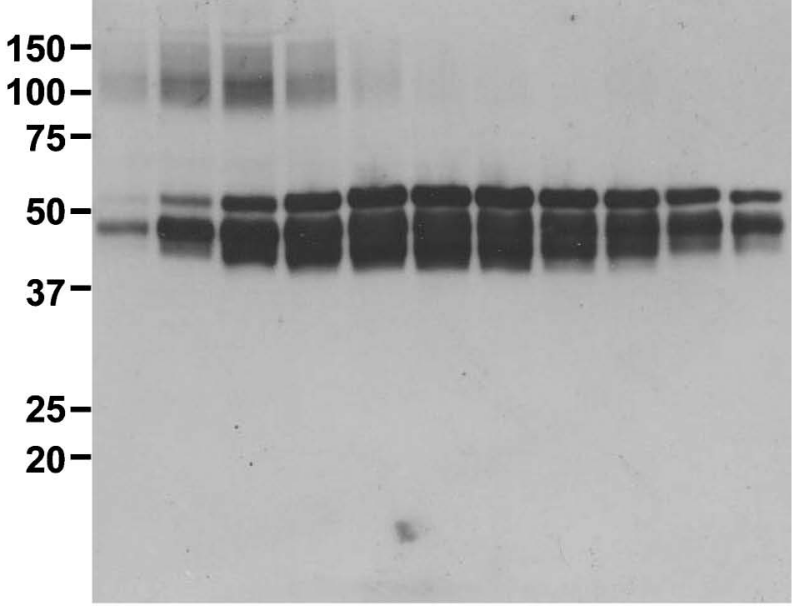

Fig. 2. Separation of Asialofetuin from Higher-molecular Weight Proteins

Commercially available fetuin (prepared by the Spiro method) treated with sialidase was applied to a Sephadex G-75 gel filtration column equilibrated with EDTA-PBS. (a) Elution profile of asialofetuin. Fractions $(2 \mathrm{ml}$ each) were collected and protein concentrations measured by absorbance at $280 \mathrm{~nm}$. Fractions 23-33 (numbered in the elution profile) were subjected to western blotting. (b) Analysis of fractions 23-33 by western blotting using polyclonal antiserum raised against fetuin. These fractions were subjected to SDS-PAGE and western blotting. Immunoreactive bands were visualized using anti-fetuin antiserum. The numbers across the top of the gel correspond to the eluant fraction numbers from the Sephadex G-75 column in (a). 
hinder identification of the crosslinked products between the galectins and asialofetuin, asialofetuin was prepared from commercially available bovine fetuin. No small fragments resulting from peptide bond cleavage were observed after treating commercially available bovine fetuin with sialidase. Since some higher-molecular weight proteins were present in this fraction, the reaction mixture was applied to a Sephadex G-75 gel filtration column in EDTA-PBS. The elution of the glycoproteins was monitored by measuring the absorbance of each fraction at $280 \mathrm{~nm}$. As shown in Fig. 2 (a), two protein peaks were observed, one at fraction 25 and the other at fraction 27 . The former peak contained proteins with high molecular weights between 100 and $150 \mathrm{kDa}$, as well as asialofetuin with molecular weights in the range of 50-60 kDa (Fig. 2(b)). Crosslinking of asialofetuin, used as model glycoprotein ligand, to galectins would result in high-molecular weight crosslinked heterodimers spanning this protein molecular weight range. Thus the 100 - to $150-\mathrm{kDa}$ contaminant proteins would complicate detection of the crosslinked product by western blotting. Therefore fractions 28-33, which contained only asialofetuin, were collected and further purified on an affinity column containing immobilized nematode galectin LEC-1.

Purified asialofetuin (containing no small frag- ments or high molecular weight proteins) was crosslinked using the photoactivated crosslinker BPM. Photoactivated crosslinking of the three Gal-1 forms with asialofetuin was carried out using BPM as described previously. To test whether these three Cyscontaining mutants of Gal-1 can result in a crosslinked product with asialofetuin via the crosslinker BPM, proteins in the reaction mixture were subjected to western blotting using antibodies raised against Gal-1 and fetuin. As shown in Fig. 3, an additional major band was detected by western blotting using both anti-Gal-1 and anti-fetuin antibodies, consistent with the formation of a covalently crosslinked complex of K28C Gal-1 and asialofetuin only in the presence of BPM. ${ }^{14)}$ Although both the A27C and S29C forms of Gal-1 had the ability to bind asialofetuin, as shown in Fig. 1, no crosslinked product was observed for either of these recombinant proteins. $\mathrm{Ala}^{27}$ and $\mathrm{Ser}^{29}$ flank Lys ${ }^{28}$ in the $\beta$-strand of Gal-1, and the side chain of the Ala and Ser is oriented on the opposite side of the $\beta$-sheet from the $\mathrm{Lys}^{28}$ side chain. ${ }^{15)}$ Therefore the sulfhydryl portion of the side chain of the cysteine residue introduced in place of $\mathrm{Ala}^{27}$ or $\mathrm{Ser}^{29}$ would be oriented on the opposite side of the $\beta$-sheet as the $\mathrm{K} 28 \mathrm{C}$ mutant. Thus the orientation of the side chain of the amino acid for substitution by cysteine is important for successful crosslink-
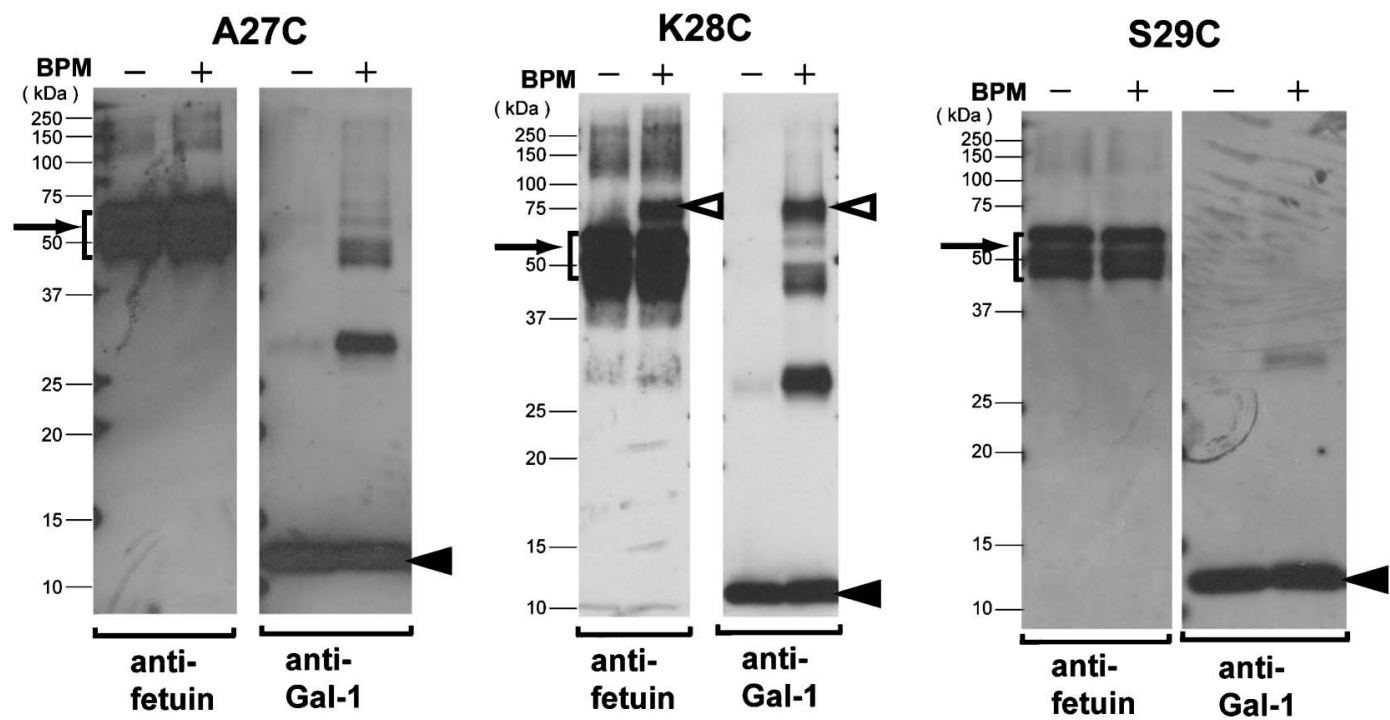

Fig. 3. Crosslinking Reaction of A27C, K28C, and S29C Gal-1 Mutants and Asialofetuin Using BPM

Photoactivated crosslinking of the recombinant A27C, K28C, and S29C forms of Gal-1 to asialofetuin were carried out in the presence $(+)$ or absence $(-)$ of BPM in the dark followed by irradiation with ultraviolet light in the presence of purified asialofetuin. The proteins were then separated by SDS-PAGE and examined by western blotting with antiserum against either Gal-1 or fetuin. An additional band with a molecular mass of approximately $75 \mathrm{kDa}$, indicated by a white arrowhead, was detected for the K28C Gal-1 mutant using both anti-Gal-1 and anti-fetuin antibodies, but was not detected for the A27C or S29C Gal-1 mutants. The black arrowheads show the position of Gal-1 mutants, and the positions of asialofetuin are shown by brackets. Molecular weight markers are indicated on the left of each panel. 
ing of galectin to its bound glycoprotein ligand using BPM. This approach should be very useful for identifying residues in other galectins suitable for cysteine mutagenesis, allowing identification of endogenous ligands for each galectin by crosslinking using BPM.

Acknowledgements This study was supported in part by a Grant-in-Aid for scientific research (No. 15790048) from the Ministry of Education, Science, Sports, and Culture of Japan. We thank Drs. Yoko Nemoto-Sasaki and Tomoharu Takeuchi for helpful discussions.

\section{REFERENCES}

1) Kasai K., Hirabayashi J., J. Biochem. (Tokyo), 119, 1-8 (1996).

2) Leffler H., Carlsson S., Hedlund M., Qian Y., Poirier F., Glycoconj. J., 19, 433-440 (2004).

3) Cooper D. N., Barondes S. H., Glycobiology, 9, 979-984 (1999).

4) Barondes S. H., Castronovo V., Cooper D. N. W., Cummings R. D., Drickamer K., Feizi T., Gitt M. A., Hirabayashi J., Hughes C., Kasai K., Leffler H., Liu, F.-T. Lotan R., Mercurio A. M., Monsigny M., Pillai S., Poirer F., Raz A., Rigby P. W. J., Rini J. M., Wang J. L., Cell, 76, 597-598 (1994).

5) Liu F. T., Rabinovich G. A., Nat. Rev. Cancer, 5, 29-41 (2005).

6) Hernandez J. D., Baum L. G., Glycobiology, 12, 127R-136R (2002).

7) Garner O. B., Baum L. G., Biochem. Soc. Trans., 36, 1472-1477 (2008).
8) Hirabayashi J., Hashidate T., Arata Y., Nishi N., Nakamura T., Hirashima M., Urashima T., Oka T., Futai M., Müller W. E. G., Yagi F., Kasai K., Biochim. Biophys. Acta, 1572, 232-254 (2002).

9) Stowell S. R., Arthur C. M., Mehta P., Slanina K. A., Blixt O., Leffler H., Smith D. F., Cummings R. D., J. Biol. Chem., 283, 1010910123 (2008).

10) Arata Y., Tamura M., Nonaka T., Kasai K., Biochem. Biophys. Res. Commun., 350, 185190 (2006).

11) Dormán G., Prestwich G. D., Biochemistry, 33, 5661-5673 (1994)

12) Arata Y., Baleja J. D., Forgac M., J. Biol. Chem., 277, 3357-3363 (2002).

13) Arata Y., Baleja J. D., Forgac M., Biochemistry, 41, 11301-11307 (2002).

14) Tamura M., Igarashi T., Kasai K., Arata Y., Biochem. Biophys. Res. Commun., 390, 581584 (2009).

15) López-Lucendo M. F., Solís D., André S., Hirabayashi J., Kasai K., Kaltner H., Gabius H.-J., Romero A., J. Mol. Biol., 343, 957-970 (2004).

16) Hirabayashi J., Kawasaki H., Suzuki K., Kasai K., J. Biochem. (Tokyo), 101, 987-995 (1987)

17) Arata Y., Hirabayashi J., Kasai K., J. Biochem. (Tokyo), 121, 1002-1009 (1997).

18) De Waard A., Hickman S., Kornfeld S., J. Biol. Chem., 251, 7581-7587 (1976). 